\title{
Development and validation of two food portion photograph books to assess dietary intake among adults and children in Central Africa
}

\author{
Norbert Amougou $^{1 *}$, Emmanuel Cohen ${ }^{1,2}$, Marie L. Mbala ${ }^{1}$, Basile Grosdidier ${ }^{1}$, Jonathan Y. Bernard ${ }^{1}$, \\ Rihlat Saïd-Mohamed ${ }^{1,3}$ and Patrick Pasquet ${ }^{1}$ \\ ${ }^{1}$ Muséum National d'Histoire Naturelle, Centre National de la Recherche Scientifique, Université Paris 7 Diderot, Sorbonne Paris Cite, \\ Sorbonne Universités, Unité Eco-Anthropologie et Ethnobiologie (UMR 7206), Musée de l, 17 place du Trocadéro, 75016 Paris, France \\ ${ }^{2}$ Centre National de la Recherche Scientifique, UMI 3189 'Environnement, Santé, Société', Faculté de Médecine Secteur \\ Nord 51, Bd Pierre Dramard, 13344 Marseille Cedex 15, France \\ ${ }^{3}$ Medical Research Council/Wits Developmental Pathways for Health Research Unit, Department of Paediatrics and Child \\ Health, Faculty of Health Sciences, University of the Witwatersrand, 7 York Road, Parktown 2193, Johannesburg, South Africa \\ (Submitted 16 March 2015 - Final revision received 22 October 2015 - Accepted 26 November 2015)
}

\begin{abstract}
Owing to nutritional transition in Cameroon, one in two adults is overweight and one in five is obese, and $8.1 \%$ of children are overweight and $2 \cdot 1 \%$ are obese. Given this phenomenon, dietary intake assessment is needed to establish appropriate preventive nutrition-sensitive strategies. Our aim was to develop and test the validity of two food portion photograph books (FPPB) to be used as visual aids for adults and children taking part in a 24-h dietary recall. To design FPPB, interviews and focus group discussions were undertaken with women to obtain consensus on the local categorisation of foods. For each cooked and weighed food, three photographs of the average small, medium and large serving portion sizes were taken, and four intermediary portion sizes were calculated. To validate the FPPB, a sample of adults (361) and children (224) were asked, at meal times, to self-serve a food portion prepared in the household and the portion sizes were weighed; $24 \mathrm{~h}$ after the measurement, the same subjects were shown the appropriate FPPB and were asked to indicate the food and the portion they consumed. In adults, of the 821 portions tested, $77 \%$ were accurately estimated, whereas in children $74 \%$ of the 556 portions tested were accurately estimated. For both groups, the small- and medium-sized portions were frequently selected and accurately estimated ( $>70 \%)$. Our findings suggest that the adult and children's FPPB can be used in Cameroon to estimate food portion sizes, and thus nutritional intake in the frame of the 24-h dietary recall.
\end{abstract}

Key words: Dietary intakes: Portion size photographs: Food energy density: Central Africa

Most countries in sub-Saharan Africa are experiencing marked demographic changes, characterised by the migration of the populations from rural to urban areas, and Cameroon is no exception. At the same time, urban populations of the subcontinent are experiencing the so-called 'nutritional transition', which is characterised by major changes in the lifestyle and dietary habits associated with the development of chronic and degenerative diseases ${ }^{(1)}$. In Cameroon, the landscape between the latitudes $2^{\circ}$ and $13^{\circ} \mathrm{N}$ represents the first meeting point between Bantu-language and Sudanese-language groups and is marked by three distinct habitats: equatorial forest in the southern and central regions and savanna in the northern Sahelian regions ${ }^{(2)}$. In the past, this environmental heterogeneity led to the development of two different subsistence strategies: tropical vege-cultural systems (yam and palm oil), which developed in the forests of southern and central Cameroon, and cereal cultivation (sorghum and millet) in the northern regions ${ }^{(3)}$. Nowadays, nutritional transition, combined with poverty, makes some traditional food items unaffordable to many, and consequently the Cameroonians switch from the traditional staple food to high-fat and high-sugar content foods ${ }^{(4,5)}$. Two recent studies reported that half of the adults are overweight and one in five is obese ${ }^{(6,7)}$. In addition, over the period 1975-2000, the prevalence of overweight in children doubled and the prevalence of obese children quadrupled ${ }^{(5,8,9)}$. Given the magnitude of this phenomenon, the nutritional anthropology for Central African migrants to urban areas project (ANTRAC) was initiated to identify and describe the determinants of non-communicable diseases in populations in transition living in the Central Africa region (Cameroon and Gabon). Ultimately, the results of the ANTRAC project will inform public health strategies for the prevention of overweight and obesity in Central Africa.

An objective of the ANTRAC project was to describe and assess the dietary intake of the populations living in Cameroon and Gabon. Research studies indicate that the quantity of food consumed is positively correlated with the energy intake $e^{(10-13)}$.

Abbreviation: FPPB, food portion photograph books.

* Corresponding author: N. Amougou, email namougou@mnhn.fr 
Therefore, estimating the portion size is considered a key factor in the analysis of nutritional intake ${ }^{(14,15)}$. Several assessment tools in the form of books with photographs of food portions have been developed and used in dietary assessment studies to enhance the ability of the participants to accurately report the amounts of food items consumed ${ }^{(15-17)}$. The number and sizes of photographs used to depict food portions varied among studies. Livingstone \& Robson ${ }^{(18)}$ using single portion size colour photographs reported that it may be a useful tool, even though some of the food photographs were more effective than others in helping subjects to accurately describe the amounts consumed. The authors concluded that increasing the number of photographs to four or more per food item may help improve the accuracy of responses. More recently, studies reported that the use of a series of three photographs and the intermediate unrepresented (virtual) portion sizes is associated with relatively small errors ${ }^{(16,17)}$. These findings support the validity of using food photographs as a tool for quantifying food portion size in epidemiological dietary surveys on different age groups.

However, it is well known that perception, conceptualisation and memory affect the report of food portion size ${ }^{(19)}$. Perception is the ability to relate the amount of food present in reality to the amount presented by a portion size aid. Conceptualisation is the ability to develop a mental picture of a food portion not actually present and to relate it to a portion size aid. Memory is the ability to accurately recall the amount of food eaten. These three dimensions can therefore be affected by the age of the subject ${ }^{(20)}$, the use or not of household objects during the test ${ }^{(21)}$ and of course by the number of different food portion sizes, their placement in the food photograph book and the dimensions of each photograph ${ }^{(17)}$. Indeed, children's cognitive abilities have been shown to be relatively underdeveloped, and thus they may be less accurate than adults when recalling dietary intake and food portions ${ }^{(20,22,23)}$. In addition, the use of household objects (e.g. plate, cup, spoons) has been recommended in a number of nutrition education and consumer publications to help individuals estimate the food portion sizes ${ }^{(21,24)}$. However, many studies still use inappropriate estimation tools and methods to assess the accuracy of portion size reports. Indeed, most studies assess the accuracy of their tool by evaluating either perception only or perception combined with conceptualisation, whereas little is known about evaluation of all the three factors of errors in reporting food portion size (perception, conceptualisation and memory) $)^{(15,17)}$

As part of a larger experimental programme studying the determinants of non-communicable diseases for Central Africans in transition, the objective of our present study was to develop and validate two local food portion photograph books (FPPB), respectively, for adults and children living in Cameroon, to be used as visual aids during the 24-h dietary recall that is usually used in nutritional surveys.

\section{Methods}

\section{Study design}

The study was undertaken in the capital city of Cameroon, Yaoundé, which includes more than 2.5 million inhabitants living in $297 \mathrm{~km}^{2}$. Similar to other parts of Cameroon, there is a vast disparity in income in the city of Yaoundé ${ }^{(4)}$. Given that there were no census data available for Yaoundé at the time of the study, we used the representative household surveys previously carried out by the National Institute of Statistics of Cameroon to proportionally determine the number of households per district and the proportion of each major ethnic group that should be included in the study to ensure socio-economic and ethnic representativeness. However, owing to the lack of settlement maps for Yaoundé districts, we had to randomly recruit households by carrying out door-to-door fieldwork in the different districts. After being informed about the objective of the study, approval and written consents were obtained from the chief of each household before the start of the study. The present study was conducted according to the guidelines laid down in the Declaration of Helsinki, and permission to carry out the study and ethical clearance were obtained from the ethical committee of the Ministry of Public Health of Cameroon. In all, 585 healthy participants aged between 3 and 84 years were recruited.

\section{Development of adults and children food portion photograph books}

Preliminary studies were conducted with households, vendors and restaurants in Yaoundé to determine the commonly consumed meals and preparation methods in urban areas. This study was based on interviews with the women in charge of preparing meals for the household and, in the case of restaurants, with the chef. These were followed by focus group discussions to obtain consensus on the categorisation of foods. In these studies, it was found that Cameroonian food can be grouped into what is locally named 'sauce' (vegetables green leaves, seeds and fruits), cereal (rice, pasta and maize), sandwich (bread with butter, chocolate or meat), meat (chicken, beef and pork), fish (mackerel, carp, sardines and cod), leguminous seeds (peas and beans), what is named 'complement' (cassava, plantain, cocoyam, banana, potatoes and their transformed products), a stew called 'malaxé' (mixed food), sweet (candies, chocolate and biscuits) and beverage (soft drinks, beer, wine, whisky and fruits juice) (Table 1).

The methodology used to develop the FPPB was the following. At home or at restaurants, once the reported meal was cooked by the mother or the chef, the mother or the server served the food as usual to each participant. For adults and for children, all the portions served for each food item were then weighed using electronic kitchen scales (Compact Scale Ohaus $5000^{\circledR}$; Sigma Aldrich). For each food item, the medium portion size ' $\mathrm{D}$ ' was determined as the mean of the weight measured for the food item, for adults and for children. Thereafter, according to the three sigma rule ${ }^{(25)}$, the large portion size ' $\mathrm{F}$ ' was taken as the mean +1 SD and the small portion ' $\mathrm{B}$ ' was taken as the mean -1 SD. In addition, intermediate portions ' $\mathrm{A}$ ' and ' $\mathrm{G}$ ' were taken as the portions located before and after the small and large portions, respectively, whereas ' $\mathrm{C}$ ' and ' $\mathrm{E}$ ' were located before and after the medium portion, respectively. Therefore, the 'A' and ' $\mathrm{C}$ ' portions were taken as the medium portion weight -1.5 and $0.5 \mathrm{sD}$, respectively, whereas the ' $\mathrm{E}$ ' and ' $\mathrm{G}$ ' portions were taken as the medium portion weight +0.5 and $1.5 \mathrm{sD}$, respectively. 


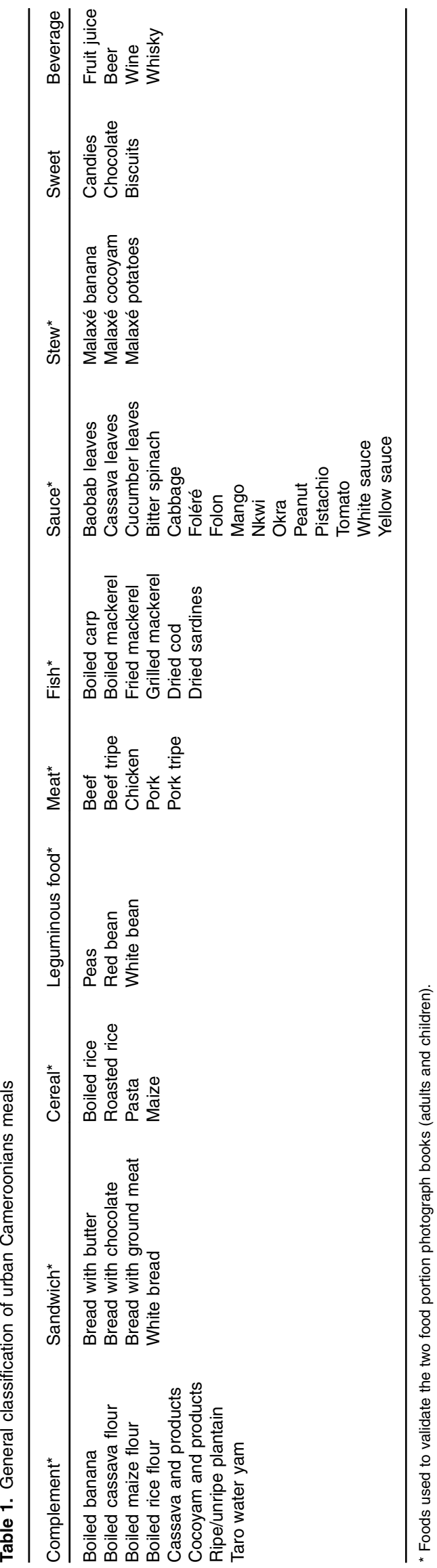

Photographs were then taken and depicted on a standard deep white plate for the small ' $\mathrm{B}$ ', medium ' $\mathrm{D}$ ' and large ' $\mathrm{F}$ ' portions of each food item using a Nikon camera (DX AF-S Nikkor 18-70 mm; Nikon), whereas the 'A', 'C', 'E' and 'G' portions were considered as virtual. A knife and a fork were included as reference items. The background was a light cream-coloured sheet, and two Brushed Steel Mainstays 35-W Halogen Desk Lamps (Walmart Mainstays) were used to better show the volume of the foods on the plate. The focal length of the camera lens was set at $50 \mathrm{~mm}$, and the photographs were taken at an angle of $55^{\circ}$ to the horizontal to obtain a result as close as possible to the human sight when seated at the table. To obtain pictures as close as possible to real life, the prints were enlarged by colour photocopying to $75 \times 100-\mathrm{mm}$ size. For processed foods such as sweets (candies, chocolates and biscuits) or beverages (soft drinks, beer, wine, whisky and fruits juice), information about the weight for each item was obtained from the labelling. A total of 1033 food portions were weighed -764 for adults and 269 for children.

\section{Validation procedure of the adult and child food portion photograph books}

The validation procedure was undertaken using the ageappropriate FPPB tool for adults and children during lunch or dinner in the households in Yaoundé.

Adults and 8-13-year-old children were told that they would eat meals as usual in the presence of a trained research assistant on the 1st day, and then respond to some questions about the food they eat on the 2nd day. For 3-7-year-old children, parents were surrogate reporters of their children's food intake. They were not informed that they would be asked about portion sizes consumed. To approximate real-life eating, the mother of the family served the meal in household containers to each member of the family rather than in standard portions on a standard plate for each food. A trained research assistant ensured that no foods were spilled, exchanged or thrown out. Each serving container was weighed using an electronic kitchen scale before and after serving the food. Any remaining food on the plate was also weighed to determine the amount consumed.

On the 2nd day, photographs of the FPPB were shown to the participants (or parents of the children) to enable them to visualise three images of each food and select the portion sizes that they estimated to be the closest to the amount that they had eaten the day before (24-h recall). Participants were told that they could also indicate whether a portion was smaller or larger than the images of the food portions. Thus, for each food presentation (Fig. 1), participants were offered the option to select 'A', 'B', 'C', 'D', 'E', 'F' and ' $G$ ' to best represent what they had consumed the previous day. The responses were ranked as 'correct' if the estimated weight was within $10 \%$ of the real weight measured by the trained fieldworker the day before, overestimated if the estimated weight was $>10 \%$ of the real weight and underestimated if the estimated weight was $<10 \%$ of the real weight.

\section{Data treatment and analysis}

Frequency distributions and descriptive statistics (mean values and standard deviations and 95\% CI) were used to describe 

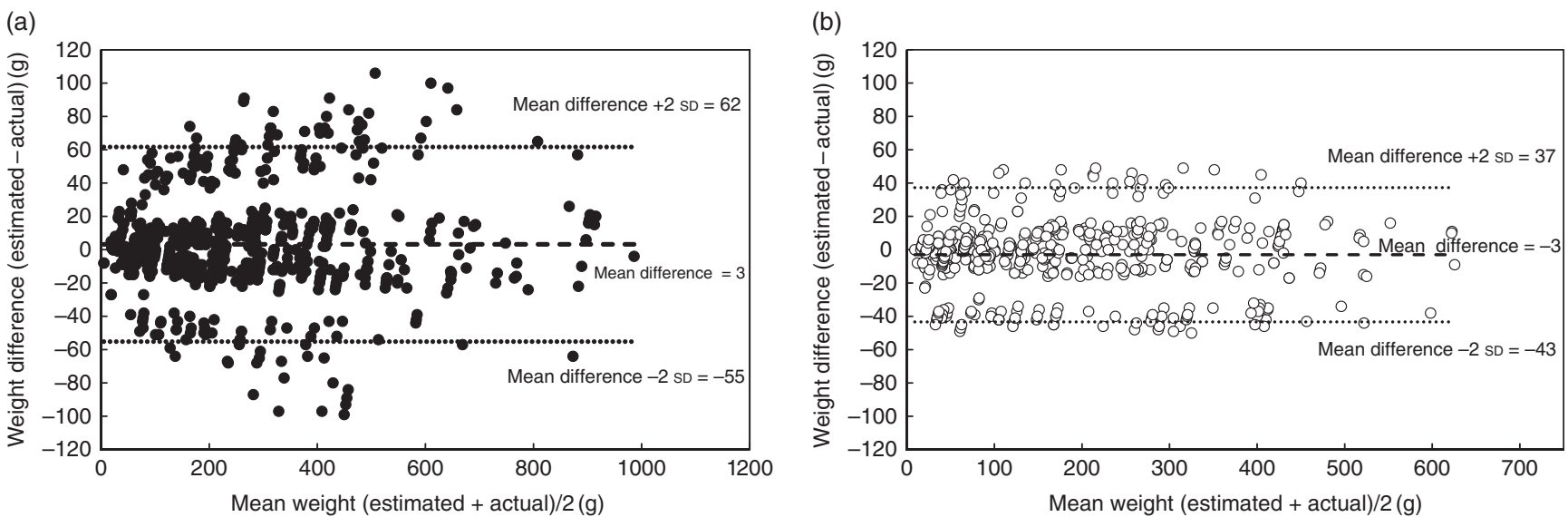

Fig. 1. Plots of the difference between estimated weight and actual weight against the means of weight obtained by estimated and actual portions for adults (a) and children (b) and for all foods. The plots also show the mean difference of estimated weight and actual weight (the bias) along with the mean difference \pm 2 sD ( $95 \%$ limits of agreement).

the characteristics of the sample and the responses of adults and children.

The number and percentage of portions correctly identified, overestimated and underestimated were calculated for adults and children and for every portion of each food item. Bland-Altman regression ${ }^{(26)}$ was used to measure the agreement between estimated and consumed portions; the $95 \%$ limit of agreement was calculated.

The number of correct, overestimated and underestimated portions was compared for age (adults and children) and sex groups using one-way ANOVA. The statistical analyses were carried out using R 2.9.2 software.

\section{Results}

\section{Demographics of participants}

A total of 585 participants were included in this study, including 361 adults (193 women and 168 men) and 224 children (129 girls and ninety-five boys). The age of adults ranged from 14 to 84 years with average ages of 24 (SD 7) and 30 (SD 15) years for men and women, respectively. For children, the age ranged from 3 to 13 years and the average age was 8 (SD 3) years for both girls and boys.

\section{Pattern of responses from adults}

As there were no significant sex-related differences in the ability to estimate portion size using the adult FPPB, data are presented for the whole group (Table 2).

Of the total of 821 responses, 635 were correct, 114 were overestimated and seventy-two were underestimated. Clearly defined solid food showed the highest numbers of correct responses and ranked as follows: sandwich $>$ meat $>$ complement. The poorest responses were obtained for sauce and leguminous foods, which are amorphous foods. For semi-solid foods, the following rank was obtained: cereal $>$ stew $>$ fish. Therefore, the number of correct responses for solid food was significantly $(P \leq 0.05)$ higher than that for the amorphous type.
Stew, sauce and meat are the foods most frequently overestimated and represented 21, 18 and 16\%, respectively, whereas leguminous food, cereal and fish were the foods frequently underestimated (Table 2). Amorphous foods were overestimated and underestimated to the same level, whereas for solid foods there were significantly more overestimates than underestimates of portion size $(P \leq 0 \cdot 05)$.

Complements and sauces were more frequently consumed than the other food items. The frequencies of selection of photographs $v$. virtual portion size are as follows: A (17\%), B (27\%), C (14\%), D (21\%), E (9\%), F (9\%) and G (3\%). Photographs of the portions (B, D and F) represent $57 \%$ of the responses, whereas $43 \%$ are for virtual portions (A, C, E and G). In contrast, the correct responses for virtual portions were relatively higher $(\mathrm{A}=83 \%, \mathrm{C}=84 \%$ and $\mathrm{E}=83 \%)$ than that for the photographed portions $(\mathrm{B}=77 \%, \mathrm{D}=68 \%$ and $\mathrm{F}=68 \%$ ) but the differences were not significantly different (Table 2). Small portions (A and B) were significantly $(P \leq 0.05)$ overestimated, whereas large portions ( $\mathrm{E}$ and $\mathrm{F}$ ) were underestimated (Table 2).

\section{Pattern of responses from children}

As no significant sex-related differences in the ability to estimate portion size using the appropriate children's FPPB were observed, data are presented for the whole group (Table 3).

Of the total of 556 responses, 413 were correct, forty-four were overestimated and ninety-nine were underestimated (Table 2). The numbers of correct responses were quite similar for solid and amorphous foods except for cereal and bread. Therefore, the following ranking was obtained: sandwich $>$ stew $>$ meat $=$ leguminous foods $>$ sauce $>$ complement $>$ fish $>$ cereal.

Fish and leguminous food/meat were the foods most frequently overestimated, and this represented 22 and $13 \%$, respectively, whereas cereal and sauce were frequently underestimated (Table 3). Solid foods were overestimated and underestimated to the same level, whereas amorphous foods were significantly $(P \leq 0.05)$ more underestimated than overestimated. 


\section{NS British Journal of Nutrition}

Table 2. Correct $(\mathrm{C})$, overestimated $(\mathrm{O})$ and underestimated $(\mathrm{U})$ adult responses for each food portion size

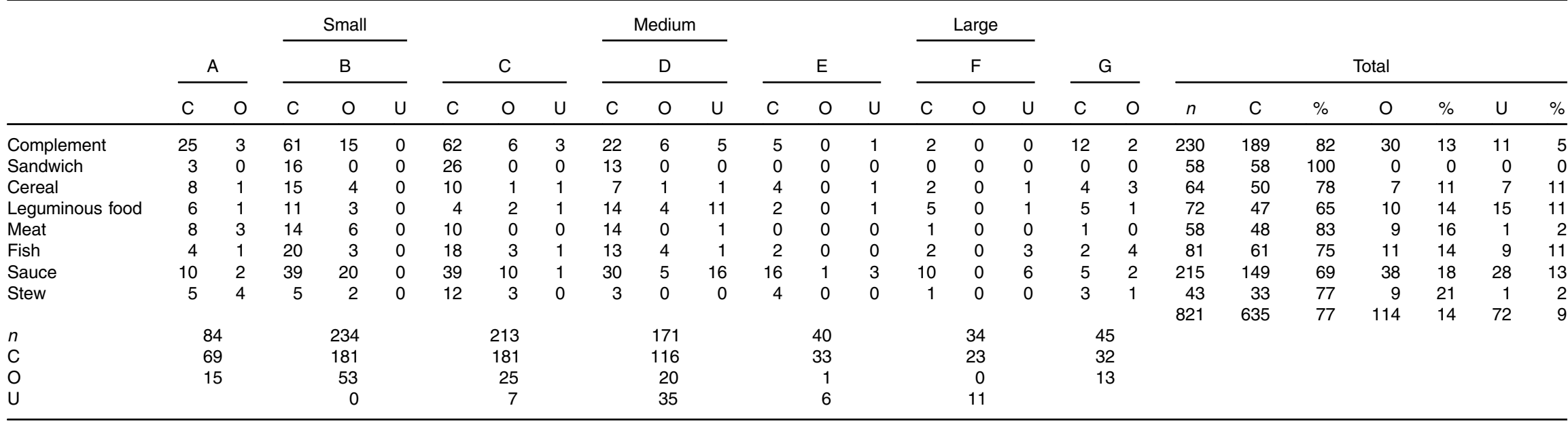

$n$, number of portions per food group; B, D and F correspond to small, medium and large photograph portions, respectively, whereas $\mathrm{A}, \mathrm{C}, \mathrm{E}$ and $\mathrm{G}$ are virtual portions.

Table 3. Correct $(C)$, overestimated $(\mathrm{O})$ and underestimated $(\mathrm{U})$ child responses for each food portion size

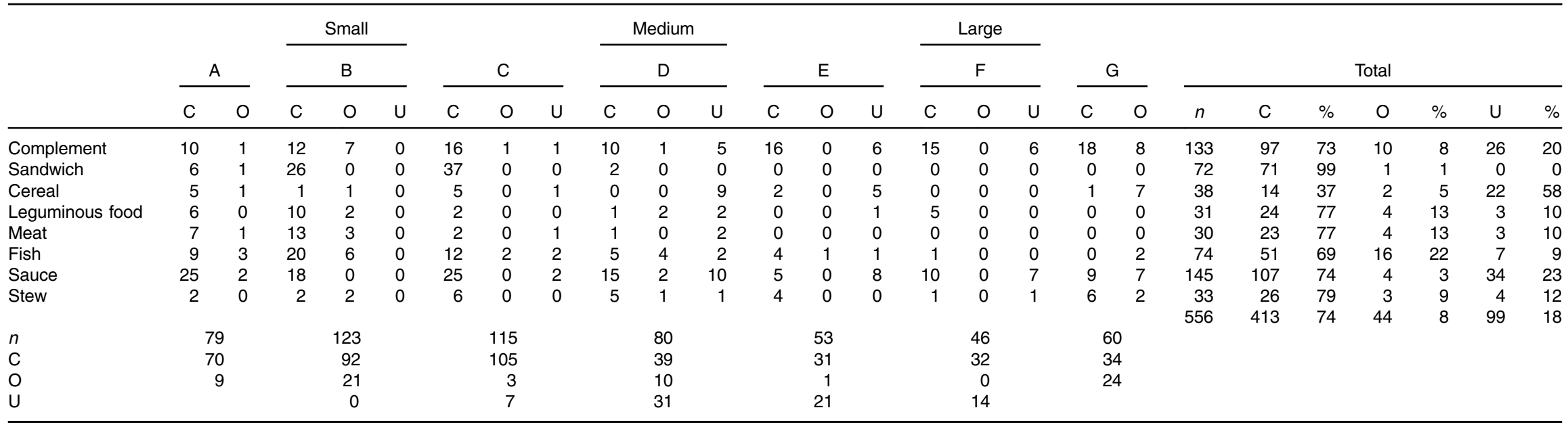

$n$, number of portion per food group; B, D and F correspond to small, medium and large photograph portions, respectively, whereas $\mathrm{A}, \mathrm{C}, \mathrm{E}$ and $\mathrm{G}$ are virtual portions. 
Complements and sauces were more frequently eaten compared with other foods. The frequencies of selection of photographs $v$. virtual portion size are as follows: A (14\%), B (22\%), C (21\%), D (14\%), E (10\%), F (8\%) and G (11\%). Therefore, photographed portions (B, D and $\mathrm{F}$ ) represent $44 \%$ of the responses, while $56 \%$ are for virtual portions (A, C, $\mathrm{E}$ and $\mathrm{G})$. Thus, the correct responses were relatively higher for virtual portions $(A=89 \%, C=90 \%$ and $G=60 \%)$ than for the photographed portions ( $\mathrm{B}=83 \%, \mathrm{D}=48 \%$ and $\mathrm{F}=70 \%$ ), but the differences are not significantly different. As expected, the smaller portions (A and B) were significantly $(P \leq 0.05)$ overestimated, whereas the larger portions ( $\mathrm{E}$ and $\mathrm{F}$ ) were underestimated (Table 3).

\section{Degree of agreement}

The degree of agreement between the mean estimated and the true weight of the portion sizes was determined using the Bland-Altman method. The Bland-Altman method calculates the mean difference between two methods of measurement (the 'bias') and the $95 \%$ limits of agreement as the mean difference \pm 2 SD. The smaller the bias and the range between these two limits, the better the agreement is. Fig. 1 shows that, for all foods, the mean difference between weights of portion sizes estimated by the FPPB and weights of eaten portions are close to 0 for adults $(3 \mathrm{~g})$ and for children $(-3 \mathrm{~g})$. In addition, the $95 \%$ limits of agreement range from -55 to -62 for adults (Fig. 1(a)) and from -43 to -37 for children (Fig. 1(b)). Therefore, the widths of the interval are 117 and eighty for adults and children, respectively. If we look at the three distinct types of foods, then for adults the width of the $95 \%$ limits of agreement range from 46 to 87 for solid foods, from 105 to 117 for semi-solid foods and from 131 to 183 for amorphous foods. For children, the width of the $95 \%$ limits of agreement range from 10 to 68 for solid foods, from 80 to 85 for semi-solid foods and from 89 to 101 for amorphous foods.

\section{Discussion}

To our knowledge, the present study is the first to develop and validate an age-appropriate estimation tool to assess food portion sizes among adults and children in Central Africa, in order to facilitate the 24-h dietary recall usually used in nutritional surveys.

The number of food items validated in this study (75\%) was not entirely representative of all the foods included in the two photograph books, owing to the large diversity of Cameroonian meals ${ }^{(27)}$. However, the food items tested are the most commonly consumed. Our results on the frequency of correct estimation of portion sizes for adults are higher (77\%) than those obtained by similar studies ${ }^{(16,28,29)}$, which reported $50-70 \%$ correct responses. For children, comparing our findings with other studies is limited by a number of issues including the use of different tools across studies for the estimation of food portion size $\mathrm{e}^{(20,30-32)}$. It is acknowledged that, before the age of 12 years, children's recall skills, ability to estimate and indicate portion size and knowledge of food are limited, which in turn constrains their ability to self-report their food intake without parental assistance $^{(18)}$. Nevertheless, in the present study, by using an age-appropriate photograph book to recall food intake, we found that $74 \%$ of the responses were correct. This is in accordance with other food portion estimation studies ${ }^{(18)}$ conducted in developing countries that have reported a significantly improved degree of accuracy when using an age-appropriate photograph book. In this study, we validated the tool by evaluating the perception, the conceptualisation and the memory aspects as well, by allowing the participants to consume meals in their households as they do in everyday life and with household objects (plate, cups and spoons). This process ensures the accuracy of the 24-h dietary recall. Validating against these three aspects (perception, conceptualisation and memory) could partly explain the discrepancy observed between our results and the literature. Indeed, previous studies have only tested either the perception or the perception combined with the conceptualisation aspects. In addition, in contrast with our study, previous studies have used the same estimation tool for both adults and children, which can induce bias.

There are no clear guidelines in the literature regarding overestimation or underestimation responses of children and adults. We found that adults tend to overestimate (14\%), whereas children tend to underestimate (18\%). Only the study of Vereecken et $a l^{(32)}$ found similar results for children, whereas, in contrast, the other studies found overestimation ${ }^{(33)}$ or both overestimation and underestimation ${ }^{(31)}$. Furthermore, both underestimation ${ }^{(28)}$ and overestimation of food servings have been reported in studies that involve adults ${ }^{(14,17)}$. These differences could be the result of numerous factors such as visual imagery (appearance of the food), usual practice (familiarity with eating the food), behaviour chaining (association with preferred food or favourite activity during a meal or day) and preference (favourite food). Whatever the cause may be, it is important to consider how the accuracy of the tool is quantified across studies to take into account the degree and acceptability of underestimation and/or overestimation of portion sizes. Baranowski \& Domel ${ }^{(33)}$ have proposed a model of how children remember and process information in which the cognitive processes involved in the recall of dietary information can be categorised as attention, perception, organisation, retention, retrieval and response formulation. In our study, we used an age-appropriate estimation tool, and this specificity brings new insight when comparing the behaviour of adults and children to cope with size portions. Indeed, using an age-appropriate estimation tool, our results suggest that adults and children employ a number of retrieval strategies during self-report of their dietary intake: visual imagery (appearance of the food) and usual practice (familiarity with eating the food).

Food shape is another important factor explaining the ability of the participant to estimate the food portion size using a food photograph book. In the present study, dishes were first structured into eight categories (complement, sandwich, cereals, leguminous food, sauces, meat, fish and stews), and then further subdivided into three distinct types (solids, semi-solids and amorphous) instead of four (snacks, sauces, complete meals and complements) as in a recent study ${ }^{(27)}$. Our hypothesis was that the accuracy of the estimation of adult and child participants would vary across these classifications because they provide 
different perceptual challenges. For adults, clearly defined solid foods were more accurately estimated (80-100\%) than amorphous foods (65-69\%). In children, there was no significant difference between the food types (69-79\%). In the literature, there is no agreement regarding the influence of food shape. Some studies ${ }^{(16,32)}$ found the best estimations for solid foods and others ${ }^{(18,29)}$ for amorphous foods. As argued by Friedman et $a l .{ }^{(20)}$, the main explanation for this disagreement could be the variability in age/cognitive development of the study samples and differences in foods used for estimating portion sizes. Indeed, visualisation of dimensions and volume and the ability to relate it to the measurement tool used to measure the food are the pre-dominant skills used by participants $^{(14,34,35)}$. It is therefore necessary, as in the present study, to use an age-appropriate estimation tool.participants.

Although there are no clear guidelines in the literature regarding the number of food portions to be presented to the subjects $^{(15,16,36)}$, it is well established that utilisation of a single portion size photograph is associated with larger errors of estimation than a series of eight photographs, particularly with regard to underestimation of large portions. In the present study, each subject was asked to quantify the consumed food in relation to one of the three photographs or in terms of virtual portions placed among those shown in the photographs for each food. Each subject could thus choose among seven portion sizes (three depicted and four virtual). The small- and medium-sized serving portions (photographs and virtual) were the most selected portions both among adults and children compared with large ones, which is in accordance with the results published by Turconi et al. ${ }^{(17)}$ and Ovaskainen et al. ${ }^{(28)}$. Moreover, small-sized serving portions (photographs and virtual) were accurately estimated $(>70 \%)$. This last result could be explained by the poverty and the fact that many people live in the same household. Thus, the higher the number of persons in the household, the higher the frequency of small portions attributed to food sharing. Poor access to food due to poverty in Cameroon has been described in several studies ${ }^{(4)}$, and the number of people in the household has been associated with intake of small food portions. For both adults and children, the smallest portion size was overestimated for all the dishes, whereas the largest portion size was underestimated. Similar results were reported by other research groups ${ }^{(16,19,29)}$, and this phenomenon is known as the 'flat slope phenomenon'. One explanation could be that in our study, none of our participants chose a portion outside the presented range, although they were given the opportunity.

In Central Africa, people consume three meals per $\mathrm{d}$ if the economic situation of the household allows it ${ }^{(37,38)}$, whereas in the present study this practice was not common. We found that meals were consumed twice a day and were mainly based on 'complement' and 'sauces' for both adults and children. In any study on dietary intakes, it is essential that the staple foods of the diet be accurately estimated. It is therefore reassuring to note that $72-75 \%$ of the children and adults were able to estimate it correctly and that the percentage of error for all portion sizes was $<27 \%$. Owing to the frequent consumption of these foods by the population, small errors in the estimation (either overestimation or underestimation) could lead to a distorted picture of food, energy and nutrient intakes of the population ${ }^{(16)}$.

In fact, the use of our age-appropriate tools as visual aid to identify portion sizes can generate a true picture of a person's energy and nutrient intake in Cameroon. It seems crucial now to provide freely available tools for 24-h dietary recalls that could be automated and self-administrated by adults and children. Indeed, as adults and children are able to correctly estimate portion sizes, these tools would enhance dietary assessments in large-scale epidemiological studies in Cameroon and may help in the design of strategies to prevent noncommunicable diseases.

The main limitation of the present study is the small sample size due to the large expenses associated with such studies. Larger numbers would have provided more statistical power.

\section{Acknowledgements}

Thanks to Pierre Sawalda and the authors are indebted to the participants of the study.

This study is part of the ANTRAC project, led by the UMR 7206 and funded by the French National Agency for Research (ANR) between 2008 and 2012 (ANR-08-BLAN-0004). ANR had no role in the design, analysis or writing of this article.

N. A. and P. P. developed the study concept and design and the protocol of the field study. N. A., E. C., M. L. M., B. G. and P. P. conducted the field study. N. A., E. C., J. Y. B., M. L. M., B. G., R. S.-M. and P. P. analysed and interpreted the data. N. A., E. C., J. Y. B., B. G. and R. S.-M. drafted the manuscript and have provided critical revisions for important intellectual content. The study was supervised by P. P. All authors have read and approved the final version of the manuscript and have agreed to its submission.

All authors have no conflicts of interest to declare.

\section{References}

1. Popkin BM (2001) The nutrition transition and obesity in the developing world. J Nutr 131, 871S-873S.

2. Spedini G, Destro-Bisol G, Mondovi S, et al. (1999) The peopling of sub-Saharan Africa: the case study of Cameroon. Am J Phys Anthropol 110, 143-162.

3. Harris DR (1976) Traditional systems of plant food production and the origins of agriculture in West Africa. In Origins of African Plant Domestication, pp. 311-356 [JR Harlan, JMJ de Wet and ABL Stemler, editors]. The Hague: Mouton.

4. Dapi LN, Janlert U, Nouedoui C, et al. (2009) Socioeconomic and gender differences in adolescents' nutritional status in urban Cameroon, Africa. Nutr Res 29, 313-319.

5. Dapi LN, Hörnell A, Janlert U, et al. (2011) Energy and nutrient intakes in relation to sex and socio-economic status among school adolescents in urban Cameroon, Africa. Public Health Nutr 14, 904-913.

6. Pasquet P, Temgoua LS, Melaman-Sego F, et al. (2003) Prevalence of overweight and obesity for urban adults in Cameroon. Ann Hum Biol 30, 551-562.

7. Pasquet $P$ (1999) Growth, maturation and nutrition transition: with special reference to urban populations in Central Africa, abstr. In 14th International Anthropological Congress of Ales 
Hrdlicka 'World Anthropology at the Turn of Centuries', Prague-Humpolec, 31 August -4 September.

8. Fezeu L, Minkoulou E, Balkau B, et al. (2006) Association between socioeconomic status and adiposity in urban Cameroon. Int J Epidemiol 35, 105-111.

9. Said-Mohamed R, Allirot X, Sobgui M, et al. (2009) Determinants of overweight associated with stunting in preschool children of Yaounde, Cameroon. Ann Hum Biol 36, 146-161.

10. McConahy KL, Smiciklas-Wright H, Birch LL, et al. (2002) Food portions are positively related to energy intake and body weight in early childhood. $J$ Pediatr 140, 340-347.

11. Fisher JO, Liu Y, Birch LL, et al. (2007) Effects of portion size and energy density on young children's intake at a meal. $\mathrm{Am} J$ Clin Nutr 86, 174-179.

12. Rolls BJ (2009) The relationship between dietary energy density and energy intake. Physiol Behav 97, 609-615.

13. Kratz M, Baars T \& Guyenet S (2013) The relationship between high-fat dairy consumption and obesity, cardiovascular, and metabolic disease. Eur J Nutr 52, 1-24.

14. Hernández T, Wilder L, Kuehn D, et al. (2006) Portion size estimation and expectation of accuracy. J Food Comp Anal 19, S14-S21.

15. Subar AF, Crafts J, Zimmerman TP, et al. (2010) Assessment of the accuracy of portion size reports using computer-based food photographs aids in the development of an automated self-administered 24-hour recall. J Am Diet Assoc 110, 55-64.

16. Venter CS, MacIntyre UE \& Vorster HH (2000) The development and testing of a food portion photograph book for use in an African population. J Hum Nutr Diet 13, 205-218.

17. Turconi G, Guarcello M, Berzolari FG, et al. (2005) An evaluation of a colour food photography atlas as a tool for quantifying food portion size in epidemiological dietary surveys. Eur J Clin Nutr 59, 923-931.

18. Livingstone MBE \& Robson PJ (2000) Measurement of dietary intake in children. Proc Nutr Soc 59, 279-293.

19. Nelson M, Atkinson M \& Darbyshire S (1996) Food photography II: use of food photographs for estimating portion size and the nutrient content of meals. Br J Nutr 76, 31-49.

20. Friedman A, Bennett TG, Barbarich BN, et al. (2012) Food portion estimation by children with obesity: the effects of estimation method and food type. J Acad Nutr Diet 112, 302-307.

21. Byrd-Bredbenner C \& Schwartz J (2004) The effect of practical portion size measurement aids on the accuracy of portion size estimates made by young adults. J Hum Nutr Diet $\mathbf{1 7}$, 351-357.

22. Livingstone MBE, Robson PJ \& Wallace JMW (2004) Issues in dietary intake assessment of children and adolescents. $\mathrm{Br} J$ Nutr 92, S213-S222.
23. Magarey A, Watson J, Golley RK, et al. (2011) Assessing dietary intake in children and adolescents: considerations and recommendations for obesity research. Int J Pediatr Obes 6, 2-11.

24. Ensle K, Lytle JG \& Collins C (2002) Getting to Know Portion Sizes. New Brunswick, NJ: Rutgers Cooperative Extension.

25. Pukelsheim F (1994) The three sigma rule. Am Stat 48, 88-91.

26. Bland JM \& Altman D (1986) Statistical methods for assessing agreement between two methods of clinical measurement. Lancet 327, 307-310.

27. Kouebou CP, Achu M, Nzali S, et al. (2013) A review of composition studies of Cameroon traditional dishes: macronutrients and minerals. Food Chem 140, 483-494.

28. Ovaskainen ML, Paturi M, Reinivuo H, et al. (2008) Accuracy in the estimation of food servings against the portions in food photographs. Eur J Clin Nutr 62, 674-681.

29. Huybregts L, Roberfroid D, Lachat C, et al. (2008) Validity of photographs for food portion estimation in a rural West African setting. Public Health Nutr 11, 581-587.

30. Matheson DM, Hanson KA, McDonald TE, et al. (2002) Validity of children's food portion estimates: a comparison of 2 measurement aids. Arch Pediatr Adolesc Med 156, 867-871.

31. Foster E, Matthews JN, Nelson M, et al. (2006) Accuracy of estimates of food portion size using food photographs - the importance of using age-appropriate tools. Public Health Nutr 9, 509-514.

32. Vereecken C, Dohogne S, Covents M, et al. (2010) How accurate are adolescents in portion-size estimation using the computer tool Young Adolescents' Nutrition Assessment on Computer (YANA-C)? Br J Nutr 103, 1844-1850.

33. Baranowski T \& Domel SB (1994) A cognitive model of children's reporting of food intake. Am J Clin Nutr 59, 212S-217S.

34. Foster E, Adamson AJ, Anderson AS, et al. (2009) Estimation of portion size in children's dietary assessment: lessons learnt. Eur J Clin Nutr 63, S45-S49.

35. Frobisher C \& Maxwell SM (2003) The estimation of food portion sizes: a comparison between using descriptions of portion sizes and a photographic food atlas by children and adults. J Hum Nutr Diet 16, 181-188.

36. Lucas F, Niravong M, Villeminot S, et al. (1995) Estimation of food portion size using photographs: validity, strengths, weaknesses and recommendations. J Hum Nutr Diet 8, 65-74.

37. Dapi NL, Janlert U, Nouedoui C, et al. (2005) Adolescents' food habits and nutritional status in urban and rural areas in Cameroon, Africa. Scand J Nutr 49, 151-158.

38. Sharma S, Mbanya CJ, Cruickshank K, et al. (2007) Nutritional composition of commonly consumed composite dishes from the Central Province of Cameroon. Int J Food Sci Nutr 58, 475-485. 